Man versetzt $25 \mathrm{ml}$ der Chloridlösung mit 5 Tropfen einer 2\% igen wäßrigen Lösung von Säureviolett, gibt tropfenweise Salpetersäure bis zur rein blauen Farbe zu und titriert mit 0,1 n $\mathrm{AgNO}_{3}$-Lösung. Vor dem Äquivalenzpunkt ändert sich die Farbe der Lösung in Blaugrün, im Äquivalenzpunkt tritt scharfer Umschlag nach Violett ein. Soweit bisher festgestellt wurde, stören fremde Kationen und Anionen nicht. Das Verfahren dürfte sich für viele praktische Zwecke eignen, es macht die Rücktitration mit Rhodanid überflüssig.

A. KURtenacker.

Über die Wirkungsweise von Adsorptionsindicatoren stellen E. ScHULEK und E. $P \cup N G O R^{1}$ eingehende theoretische Betrachtungen an, und zwar an Hand der Farbänderung des Reaktionsgemisches, das entsteht, wenn p-Äthoxychrysoidin als Adsorptionsindicator beim. Titrieren von Silberlösungen mit Halogenidlösungen oder umgekehrt verwendet wird. Die Farbe der Farbstoffadsorbate hängt von verschiedenen Umständen, insbesondere auch vom $\mathbf{p}_{\mathrm{H}}$-Wert der Lösung ab. Man kann die Adsorbate als Säure-Basenindicatoren betrachten. Dieses Verhalten zeigen besonders die Adsorbate an Silberjodid, wo im Äquivalenzpunkt eine sprunghafte Farbänderung und eine damit verbundene $p_{\mathrm{H}}$ - ̈̈nderung zu beobachten ist ${ }^{2}$.

\title{
A. KuRTENACKER.
}

Die Titration von starken und schwachen organischen Basen in nicht wäßrigen Lösungsmitteln wie Benzol, Chlorbenzol, Nitrobenzol, Chloroform, Diäthyläther, Ligroin (Petroläther), Äthylacetat, Acetonitril und Eisessig gelingt nach J. S. FRITz ${ }^{3}$, wenn man als Maßlösung Perchlorsäure benutzt, die in Eisessig oder in einem Essigsäure-Chlorbenzol-Gemisch gelöst ist, und wenn man als Indicator Methylviolett verwendet ${ }^{4}$.

Zur Bestimmung löst man eine geeignete Menge der Base in $25-50 \mathrm{ml}$ des Lösungsmittels. Man fügt 2-3 Tropfen Indicatorlösung (Methylviolett in Chlorbenzol gelöst) hinzu und titriert mit 0,1 n Perchlorsäure-Eisessiglösung bis zum Farbumschlag nach grün. Die Perehlorsäurelösung bereitet man durch Auflösen von $8,5 \mathrm{ml} 70-72 \%$ iger Perchlorsäure in 11 Eisessig. Um Wasser zu entfernen, fügt man auf $1114 \mathrm{~g}$ Essigsäureanhydrid zu und läBt über Nacht stehen. Die Lösung wird gegen Diphenylguanidin gestellt, das nach J. CAkLToN ${ }^{5}$ präparativ gewonnen wurde.

Der Verfasser führt Beleganalysen mit Pyridin, Anilin, Benzylamin und Brucin, die in den erwähnten Lösungsmitteln gelöst waren, an. Amine mit einer Dissoziationskonstanten von mindestens $10^{-13}$ sind genau titrierbar. Sehr schwache Basen sind noch gut zu titrieren, wenn sie schwer lösliche Perchlorate bilden, wie Coffein. Dioxan, Alkohole, Aceton und größere Mengen Wasser stören. Bei den Titrationen ist auch die potentiometrische Indication verwendbar. Als Indicatorelektrode dient die Glaselektrode. Gegenelektrode ist ein Silberdraht, der mit einer dünnen Schicht von Silberchlorid bedeckt ist. Die Verwendung einer Salzbrücke erübrigt sich. Die potentiometrische Titration von Lösungen der Base in Benzol, Äther und Petroläther ist jedoch nicht möglich.

R. LANG.

Zur Bestimmung der freien Sänre in einigen Salzlösungen hat I. M. ORLowa ${ }^{6}$ Versuche angestellt. Nach Austausch der Metall-Ionen gegen Wasserstoff-Ionen an einem Kationenaustauscher (Wofatit) wurde durch Titration die Gesamtacidität

1 Analytica Chimica Acta 4, 213 (1950).

2 Vgl. E. SchuleK und E. Pungor: Analytica Chimica Acta 4, 109 (1950); vgl. diese Z. 133, 446 (1951); E. Schulek und P. Rósza: diese Z. 115, 185 (1938).

3 Analytic. Chemistry 22, 1028 (1950).

$4 \mathrm{Vgl}$. dazu auch J. S. FrItz, Analytic. Chemistry 22, 578 (1950); vgl. diese Z. 132, 197 (1951).

5 J. Amer. Chem. Soc. 44, 1469 (1922).

$6 \breve{Z}$. anal. Chim. 5, 370 (1950) [Russiseh]. 